INRA Prod. Anim., 2006, 19 (5), 357-370

\title{
L'inscription du type génétique dans les règlements techniques des produits animaux sous AOC : conséquences pour les races animales
}

\author{
A. LAMBERT-DERKIMBA ${ }^{1,2}$, F. CASABIANCA ${ }^{1}$, E. VERRIER ${ }^{2}$
}

${ }^{1}$ INRA, UR45 Recherches sur le Développement de l'Elevage, Quartier Grossetti, F-20250 Corte, France

2 INRA, INA Paris -Grignon, UMR1236 Génétique et Diversité Animales, 16 rue Claude Bernard,

F-75231 Paris, France

Courriel : lambert@inapg.fr

Les cahiers des charges d'un nombre croissant de produits animaux sous AOC imposent la présence dans les troupeaux d'une ou de quelques races seulement. Quelle part de la production animale sous AOC est concernée par un tel critère racial ? Quelle part du cheptel des races «imposées» et quelle part du cheptel des autres races assurent la production sous AOC ? Les races choisies contribuent-elles à la typicité des produits et permettent-elles d'étayer la notion d'origine, ou bien ne constituent-elles qu'un alibi marketing ?

Le marché français des produits animaux, aujourd'hui très segmenté, a vu au cours du XXème siècle l'apparition et le développement de nombreux signes de qualité ou d'origine. Les Appellations d'Origine Contrôlée (AOC) se sont principalement et précocement développées dans le secteur des produits laitiers, tout particulièrement des fromages affinés. Les AOC pour des produits carnés sont apparues plus tardivement et touchent encore un nombre restreint de produits. Depuis quelques décennies, des stratégies de développement local autour du produit rejoignent des préoccupations de mise en valeur du territoire et de maintien de la biodiversité animale domestique (Verrier et Bouffartigue 1993, Ricard 1994, Verrier et al 2005, Audiot et al 2005). Les races «locales» s'associent à des produits «locaux», et cette construction sociale tente de conforter le sens donné au mot origine dans le cadre des AOC. Les raisons du choix de race(s) animale(s) sont multiples : caractéristiques zootechniques moyennes des animaux de la race, liaison animal-milieu, image conférée au produit et atout marketing, volonté institutionnelle locale ou nationale, etc. Dans le présent article, on se propose de dresser un bilan de la liaison entre races animales et produits ani- maux AOC et d'en souligner les conséquences sur les races concernées, notamment en terme de gestion génétique ou d'orientation de leur sélection. Les fromages seront plus particulièrement mobilisés dans cette analyse pour des raisons de disponibilité des données.

\section{1 / L'évolution du contenu des cahiers des charges, les dispositions en France et dans les pays d'Europe du Sud}

\section{1 / L'évolution de la «doctrine» de I'INAO (Institut National des Appellations d'Origine)}

Le concept d'AOC est fondé sur le lien au terroir et sur la typicité : il s'appuie sur une interaction entre le milieu, l'homme et ses savoir-faire (Casabianca et al 2005). Les systèmes d'élevage associés fournissent une matière première animale orientée vers des produits issus d'usages «locaux, loyaux et constants». Autour de ces principes «fondateurs», les orientations des règlements ont été variables dans le temps (figure 1). Les premières AOC ont été construites selon une stratégie que l'on peut qualifier de défensive (Prost et al 1994) et passaient par le système juridique, la loi du 6 mai $1919^{1}$, et la jurisprudence pour se défendre contre un usurpateur (Ricard 1994). Progressivement, les syndicats de défense ont essayé de mieux définir ce que devait être leur produit. A partir des années 1980, les méthodes d'obtention des produits ont été décrites de façon précise et détaillée, l'objectif étant de souligner les liens au lieu et aux usages. Parmi les conditions de production, la race des animaux exploités est alors apparue comme un élément essentiel, citée parmi les facteurs clés dans le «canevas en 25 points» de l'INAO (CNPL-INAO 1992), mis en place au début des années 1990.

Aujourd'hui, bien que l'inscription de la race ne soit pas considérée par l'INAO comme étant un critère impératif en matière de produits animaux, les commissions d'enquête mandatées pour expertiser les demandes d'agrément continuent d'y attacher de l'importance. Dans les faits, dans les années 1970, le lien aux races animales dans le cahier des charges de certaines AOC était exprimé par le vague «recours aux

${ }^{1}$ Loi du 06/05/19, publiée au Journal officiel «Lois et Décrets» du 08/05/19, page 4726. 
Figure 1. Quelques dates de la construction historique de l'AOC.

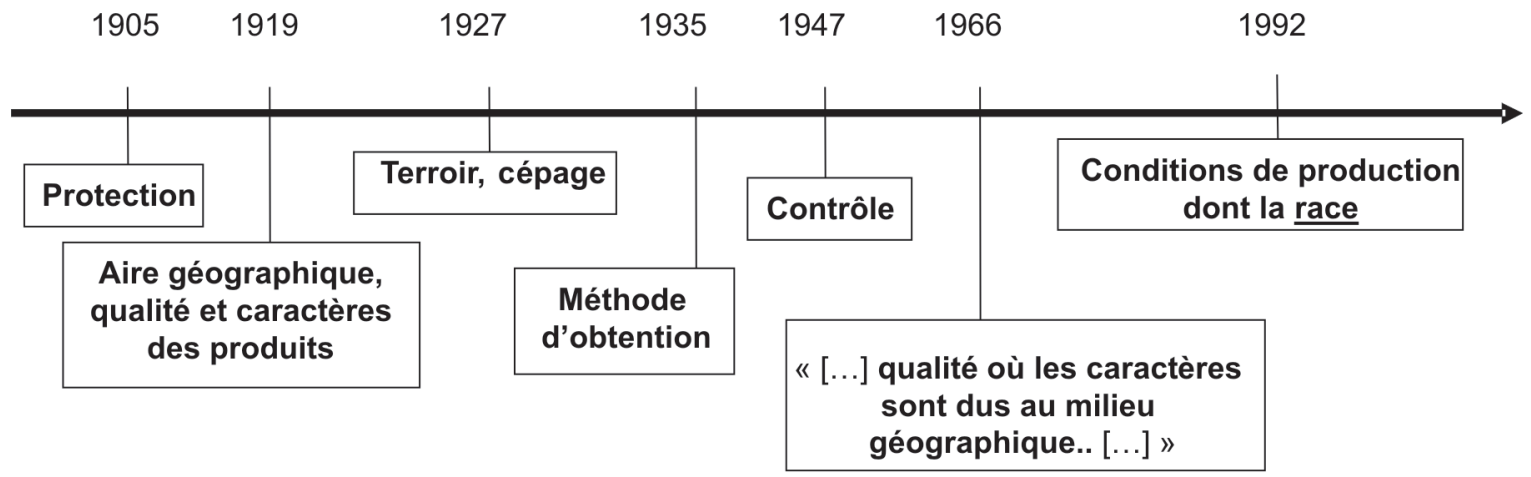

\author{
Stratégie défensive \\ "Ce que le reste n'est pas ...»
}

\section{Stratégie offensive \\ "Ce que l'AOC est ...»}

races traditionnelles». A partir des années 1990, la mention des races est toujours nominative (parfois à travers une liste positive) et, à partir des années 2000 , les nouvelles AOC sont quasiment systématiquement associées à des races (tableau 1). On a donc deux types de cas : d'une part, des AOC anciennes qui initialement ne citaient pas de races mais ont modifié ultérieurement leur décret (exemples du Laguiole ${ }^{2}$ et du Beaufort en $1986^{3}$ ) et, d'autre part, des AOC enregistrées plus récemment, qui mobilisent des races précises dès leur création (exemple de l'Abondance en $1990^{4}$, et du mouton Barèges Gavarnie en $2003^{5}$ ).

Ces évolutions peuvent être illustrées par le cas de deux AOC froma- gères, aujourd'hui de notoriété internationale et représentant les deux plus forts tonnages annuels, le Roquefort et le Comté. Le Roquefort a tout d'abord été protégé par une loi spécifique à son cas ${ }^{6}$ prévenant tout risque d'usurpation du nom Roquefort pour des produits affinés en dehors des caves du Combalou. L'obtention de l'AOC est ici basée sur la jurisprudence, comme pour le cas du Comté en 19587. Des modifications du cahier des charges sont ensuite intervenues à partir du milieu des années 1970, la plupart du temps dans le sens d'un accroissement des contraintes (figure 2). L'intégration d'un critère relatif à la race des animaux est intervenue dès $1976^{8}$ pour le Comté. A cette époque, aucun délai de mise en conformité n'avait été fixé, du fait de la large prédominance dans la zone AOC de la principale race invoquée, la Montbéliarde. En $1998^{9}$, le décret s'adapte au changement de nom de la seconde race, la Pie rouge de l'Est qui devient la Simmental française. En ce qui concerne le Roquefort, la race ovine Lacaune n'a été nommément imposée qu'en 200110. En fait, les troupeaux de la région sont quasi exclusivement constitués de brebis Lacaune mais l'ancienne référence aux races «traditionnelles» dans le décret était justifiée par l'autorisation qui, auparavant, était donnée d'importer du lait depuis d'autres bassins de production, le Pays Basque et la Corse qui disposaient de leurs races locales respectives.

Tableau 1. Evolution dans le temps de la prise en compte d'un critère de race au sein des AOC de produits animaux.

\begin{tabular}{|l|c|c|c|c|}
\hline & $\leq 1980$ & $1981-1990$ & $1991-2000$ & $2001-2005$ \\
\hline $\begin{array}{l}\text { Nombre total cumulé de produits } \\
\text { animaux sous AOC }\end{array}$ & 29 & 37 & 45 & 50 \\
\hline $\begin{array}{l}\text { Nombre total cumulé d'AOC } \\
\text { avec critère racial } \\
\text { (en \% du nombre total) }\end{array}$ & 2 & 6 & 18 & 25 \\
\hline Dont création (non cumulée) & $(6,9)$ & $(16,2)$ & $(40)$ & 6 \\
\hline Dont modification (non cumulée) & 1 & 1 & 6 & 5 \\
\hline
\end{tabular}

\footnotetext{
2 Décret du 29 décembre 1986 relatif à l'appellation d'origine contrôlée «Laguiole», publié au Journal officiel de la République française le 1 er janvier 1987.

${ }^{3}$ Décret du 29 décembre 1986 relatif à l'appellation d'origine contrôlée «Beaufort», publié au Journal officiel de la République française le 1 er janvier 1987.

4 Décret du 23 mars 1990 relatif à l'appellation d'origine contrôlée «Abondance», J. O. N 72 du 25 mars 1990.

5 Décret du 15 septembre 2003 relatif à l'appellation d'origine contrôlée «Barèges-Gavarnie», publié au Journal officiel de la République française le 20 septembre 2003 .

${ }^{6}$ Loi du 26 juillet 1925, Garantie de l'appellation d'origine du Roquefort, publiée au Journal Officiel «Lois et Décrets» du 30 juillet 1925 , page 7190.

7 Décret du 14 janvier 1958 relatif à l'aire géographique de production du gruyère de Comté ou Comté, publié au Journal Officiel «Lois et Décrets» du 18 janvier 1958 , page 720 .

8 Décret du 30 mars 1976 relatif à l'appellation d'origine «Gruyère de Comté» ou «Comté», publié au Journal Officiel «Lois et Décrets» du 13 avril 1976 , page 2245 .

9 Décret du 30 décembre 1998 relatif à l'appellation d'origine contrôlée «Comté», J.O. n 3 du 5 janvier 1999 , page 201.

10 Décret du 22 janvier 2001 relatif à l'appellation d'origine contrôlée «Roquefort», J.O. n 21 du 25 janvier 2001 , page 1283.
} 
Figure 2. Frises chronologiques de l'évolution des éléments de constitution de deux $A O C$ fromagères.

COMTÉ

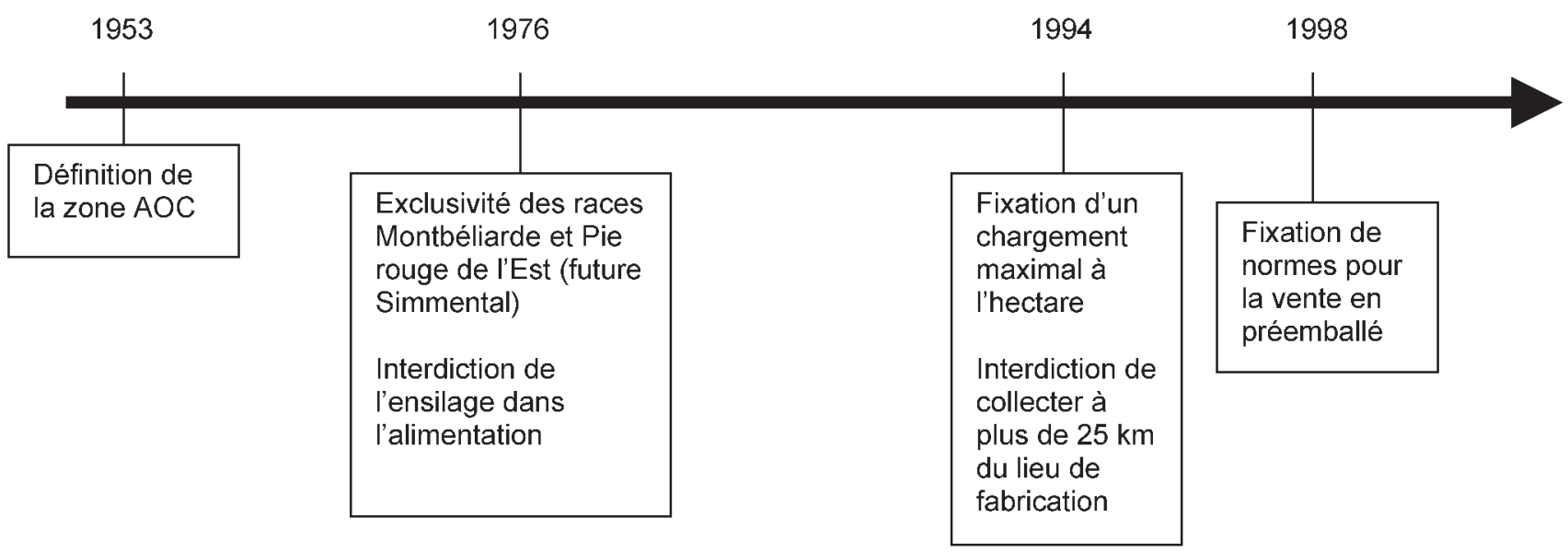

ROQUEFORT

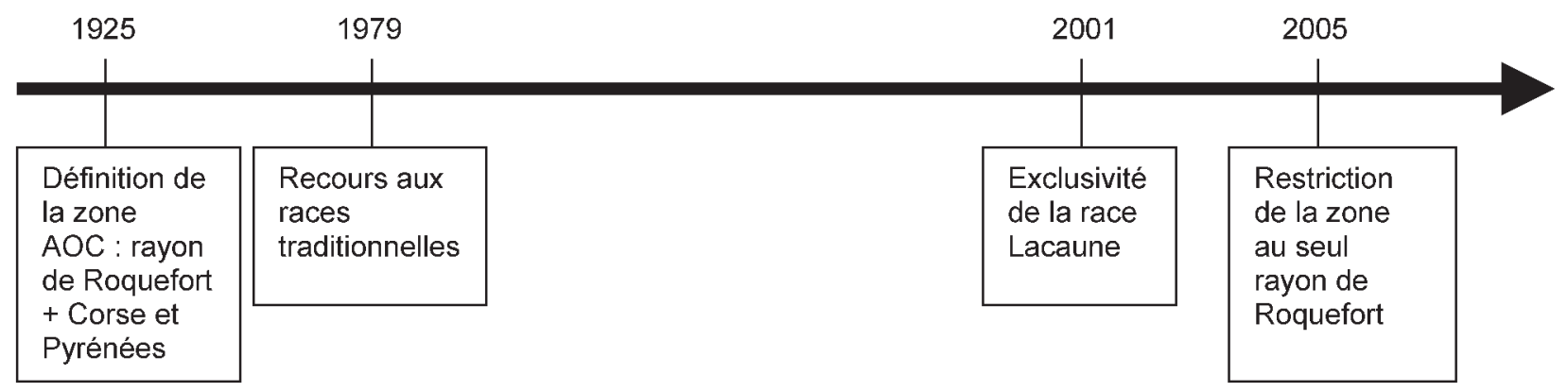

\section{2 / Le passage aux réglementa- tions communautaires}

En 1992, l’Union Européenne met en place les Appellations d'Origine Protégée (AOP). Chaque Etat membre doit faire passer ses signes nationaux en signes européens, selon diverses procédures d'harmonisation. La question se pose principalement pour la France et les autres pays de l'Europe du Sud, qui ont développé des signes d'origine selon des conceptions similaires. En France, en Espagne et en Italie, des institutions nationales ont été créées pour la gestion des produits d'origine, ce qui facilite le passage à l'harmonisation européenne. Une procédure simplifiée a été mise en place, permettant entre autres, un passage rapide des produits sous appellation d'origine (reconnaissance nationale) à un statut d'AOP (reconnaissance européenne), les dispositions définies initialement à l'échelon national pouvant être maintenues. Puis la procédure normale, pour laquelle chaque produit demandeur passe par un organisme national avant d'être pré- senté devant la commission européenne à Bruxelles, s'est instaurée en France dès 1994. La diversité des interprétations des textes dans les différents pays entraîne des différences notables dans leur application. Toutefois, les conditions de productions en lien au terroir et l'attache à une race animale ont une place importante dans les pays méditerranéens. Encore faut-il distinguer le cas des produits carnés du cas des produits laitiers. En Espagne, par exemple, une grande partie des produits charcutiers avec appellation d'origine mobilise le porc ibérique, et, au Portugal, de nombreuses AOP dans les viandes bovines intègrent un critère racial : carne Alentejana, carne Arouquesa, carne Barrosa... (Bérard et al 2004). Le cas des fromages de petits ruminants présente, en revanche, une plus faible mobilisation des races : au début des années 2000, deux fromages avec appellation d'origine sur quatre avaient un lien avec une ou des races en Espagne, trois sur neuf en France, deux sur neuf au Portugal et aucun en Italie et en Grèce (Le Jaouen et al 2001).

\section{2 / Les AOC dont le cahier des charges comprend une condition sur la race des animaux : un bilan de la situation en France}

\section{1 / Les informations disponi- bles}

Les cahiers des charges des différentes $\mathrm{AOC}$, précisant ou non la race des animaux, sont décrits officiellement sous forme de décrets, qui sont consultables sur le site de l'INAO (http://www.inao.gouv.fr/). Les statistiques de production relatives aux produits laitiers sont établies par l'INAO et sont disponibles sur la base de données du Centre National Interprofessionnel de l'Economie Laitière (CNIEL, http://www.maison-du-lait.com/). Les statistiques de production relatives aux produits carnés sont également établies par l'INAO. Nous mentionnons ici, sans le développer par la suite, l'existence d'autres types de produits ani- 
maux, le miel (deux AOC, dont le miel de Corse précise l'écotype d'abeilles), et les moules (une AOC) : en effet, dans ces cas, la notion de race ou d'écotype peut recouvrir une réalité biologique mais non une réalité sociale comme c'est le cas pour les espèces de ferme.

Lorsque plusieurs races sont autorisées pour une AOC donnée, nous avons interrogé les responsables du syndicat de défense concerné afin d'obtenir une information sur les contributions respectives de ces différentes races. Dans le cas des fromages au lait de vache ou au lait de brebis, les réponses se sont révélées cohérentes avec les statistiques locales du Recensement Général Agricole (RGA) de 2000. Si dans quelques cas (Abondance, Reblochon), des études quantifiées ont permis d'étayer ces réponses, elles demeurent en général des estimations «à dire d'experts». Pour la majorité des fromages de chèvre, en revanche, il n'a pas été possible d'obtenir d'estimation fiable : il n'en est donc pas fait état dans le présent article.

\section{2 / Les fromages AOC au lait de vache}

\section{a) Bilan global}

Parmi les 28 fromages au lait de vache bénéficiant d'une AOC, 11 comportent un critère sur la race des animaux (tableau 2). En 2004, la production avec critère racial représentait un total de 78700 tonnes, soit presque la moitié (48\%) du tonnage des fromages AOC au lait de vache. Les AOC dont le cahier des charges comporte un critère racial se situent pour l'essentiel dans les massifs du Jura et des Alpes, le Laguiole étant la seule dans le Massif Central et l'Epoisses la seule hors zone de montagne (figure 3). Aucun fromage $\mathrm{AOC}$ au lait de vache n'est associé à une race unique : lorsqu'il existe un critère racial, les cahiers des charges font référence à deux ou trois races, nommées précisément. L'AOC Salers, dans le Massif Central, n'impose pas de race animale mais, au sein de cette AOC, une mention «tradition Salers» peut être attribuée sous réserve de l'usage exclusif de vaches de race Salers.

\section{b) Analyse par région de production}

Dans ce qui semble être une logique territoriale affirmée, les AOC de Franche-Comté sont très étroitement associées à la Montbéliarde, qui est ici dans son berceau de race. Outre la Montbéliarde, la référence à la
Tableau 2. Fromages $A O C$ au lait de vache dont le cahier des charges comporte un critère sur la race des animaux et part estimée de chacune des races dans la production du lait nécessaire aux fabrications.

\begin{tabular}{|c|c|c|c|c|}
\hline Région & $A O C$ & $\begin{array}{l}\text { Production } \\
\text { en } 2004 \\
\text { (tonnes) } \\
\end{array}$ & Race(s) associée(s) & $\begin{array}{l}\text { Part de } \\
\text { chaque } \\
\text { race }(\%)\end{array}$ \\
\hline \multirow[t]{4}{*}{ Franche-Comté } & Comté & 43555 & $\begin{array}{c}\text { Montbéliarde } \\
\text { Simmental Française }\end{array}$ & $\begin{array}{c}95 \\
5 \\
\end{array}$ \\
\hline & Morbier & 6458 & $\begin{array}{c}\text { Montbéliarde } \\
\text { Simmental Française }\end{array}$ & $\begin{array}{c}95 \\
5 \\
\end{array}$ \\
\hline & Mont-d'Or & 3724 & $\begin{array}{c}\text { Montbéliarde } \\
\text { Simmental Française }\end{array}$ & $\begin{array}{c}99 \\
1 \\
\end{array}$ \\
\hline & Bleu de Gex & 516 & $\begin{array}{c}\text { Montbéliarde } \\
\text { Simmental Française }\end{array}$ & $\begin{array}{c}99 \\
1\end{array}$ \\
\hline \multirow[t]{5}{*}{ Alpes du Nord } & Reblochon & 16637 & $\begin{array}{c}\text { Abondance } \\
\text { Montbéliarde } \\
\text { Tarentaise }\end{array}$ & $\begin{array}{c}49 \\
49 \\
2 \\
\end{array}$ \\
\hline & Beaufort & 4410 & $\begin{array}{c}\text { Tarentaise } \\
\text { Abondance }\end{array}$ & $\begin{array}{l}50 \\
50\end{array}$ \\
\hline & Abondance & 1509 & $\begin{array}{c}\text { Abondance } \\
\text { Montbéliarde } \\
\text { Tarentaise } \\
\end{array}$ & $\begin{array}{c}35 \\
64 \\
1 \\
\end{array}$ \\
\hline & Tome des Bauges & 594 & $\begin{array}{c}\text { Abondance } \\
\text { Tarentaise } \\
\text { Montbéliarde }\end{array}$ & $\begin{array}{l}30 \\
20 \\
50 \\
\end{array}$ \\
\hline & Bleu du Vercors & 169 & $\begin{array}{c}\text { Montbéliarde } \\
\text { Abondance } \\
\text { Villard-de-Lans }\end{array}$ & $\begin{array}{c}80 \\
15 \\
5 \\
\end{array}$ \\
\hline Massif Central & Laguiole & 781 & $\begin{array}{c}\text { Simmental Française } \\
\text { Aubrac }\end{array}$ & $\begin{array}{l}>99 \\
<1\end{array}$ \\
\hline Bourgogne & Epoisses & 877 & $\begin{array}{c}\text { Brune } \\
\text { Montbéliarde } \\
\text { Simmental Française }\end{array}$ & $\begin{array}{l}36 \\
47 \\
17 \\
\end{array}$ \\
\hline
\end{tabular}

Sources : INAO ; Syndicats de défense AOC concernés.

Figure 3. Localisation des AOC fromagères bovines.

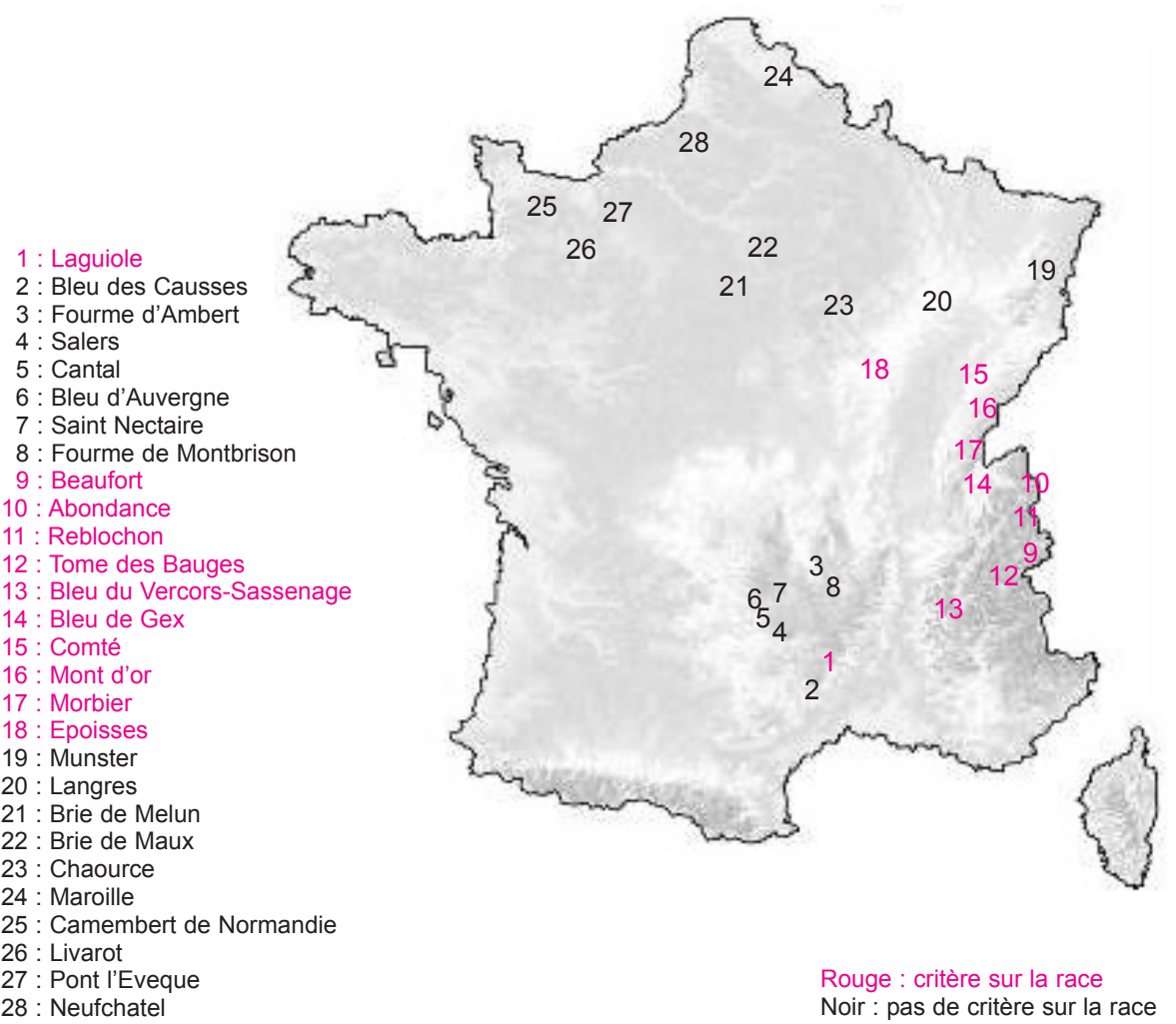


Simmental Française pour ces AOC semble motivée à la fois par la présence ancienne de cette race dans la région et par la relative proximité génétique entre ces deux races (Grosclaude et al 1990).

Dans une logique similaire (Verrier et al 2005), les AOC des Alpes du Nord incluent les deux races locales, Abondance et Tarentaise. L'une de ces AOC, le Bleu du Vercors, inclut même une race en conservation, la Villard-de-Lans. Dans le cas du Beaufort, le lien avec les deux races locales est exclusif et l'adoption d'une limite de production par troupeau confirme que l'esprit de l'AOC se situe ailleurs que dans une recherche de la productivité. Les autres AOC des Alpes du Nord incluent, en plus des races locales, la race Montbéliarde, qui du point de vue de sa contribution fait jeu égal avec ces dernières (cas du Reblochon et de la Tome des Bauges) ou les dépasse (cas de l'Abondance et du Bleu du Vercors). La place officielle de la Montbéliarde dans ces AOC semble, là encore, résulter de plusieurs motifs : implantation ancienne et importante de la race dans la région, notamment en zone de piémont; image «montagne» véhiculée par la Montbéliarde ; proximité génétique entre cette race, la race Abondance et, dans une moindre mesure, la race Tarentaise (Grosclaude et al 1990).

Dans le cas du Laguiole, le choix de l'actuelle Simmental Française s'est fait sans référence historique au terroir, alors que la race du cru, l'Aubrac, avait vu s'arrêter son exploitation à fins multiples (travail, lait, viande) au profit de l'élevage allaitant. Ce choix est en fait issu de la volonté des éleveurs adhérents de la coopérative productrice de ce fromage d'adopter une race mixte, adaptée au milieu de moyenne montagne, et présentant une composition du lait comparable à celle de vaches Aubrac traites avec amorçage et finition par le veau (Béranger et al 1970, Vissac 2001). La race Simmental étant internationale, c'est plus précisément le rameau Fleckvieh allemand (Bavière) qui a été recherché. Dès lors que les éleveurs de Simmental suisse ont procédé à une infusion massive de gènes Holstein dans leur race, alors que les éleveurs de Fleckvieh y renonçaient, cette orientation vers la Fleckvieh s'est renforcée pour l'ensemble de la Simmental Française, ce que des analyses généalogiques de la population française ont clairement révélé (Boichard et al 1996, Mattalia et al 2006). Cette constance dans le choix de la mixité des animaux s'est également traduite par l'adoption d'une limite de production dans le règlement technique. En parallèle, des actions visant à faire «renaître» un noyau de vaches Aubrac traites ont été entreprises mais il faut bien reconnaître que la contribution de ce noyau à la production demeure aujourd'hui marginale.

Quant à l'Epoisses, sa zone de production est une des deux régions d'implantation historique de la race Brune en France, race qui lui est aujourd'hui associée. La liste des races autorisées pour cette AOC semble cependant ressortir également d'un certain réalisme face à la situation en place au moment de la rédaction du cahier des charges, la seule race bien implantée dans la région à ne pas être retenue étant en définitive la Holstein.

Tableau 3. Fromages $A O C$ au lait de brebis dont le cahier des charges comporte un critère sur la race des animaux.

\begin{tabular}{|c|c|c|c|c|}
\hline Région & AOC & $\begin{array}{c}\text { Production } \\
\text { en 2004 } \\
\text { (tonnes) }\end{array}$ & $\begin{array}{c}\text { Race(s) } \\
\text { associée(s) }\end{array}$ & $\begin{array}{c}\text { Part de } \\
\text { chaque } \\
\text { race (\%) }\end{array}$ \\
\hline Massif Central & Roquefort & 18831 & Lacaune & 100 \\
\hline Corse & Brocciu & 494 & Corse & 100 \\
\hline $\begin{array}{c}\text { Pyrénées } \\
\text { Atlantiques }\end{array}$ & Ossau-Iraty & 3352 & $\begin{array}{c}\text { Basco-Béarnaise } \\
\text { Manech tête noire }\end{array}$ & $\begin{array}{c}18 \\
\text { Manech tête rousse }\end{array}$ \\
$\mathbf{5 8}$ \\
\hline
\end{tabular}

Sources : INAO ; syndicats de défense AOC concernés.

NB. Le Brocciu peut être élaboré à partir de lait de brebis ou de chèvre ; il n'est pas possible de dissocier les deux provenances et le Brocciu est ici comptabilisé parmi les AOC au lait de brebis.

Tableau 4. Fromages $A O C$ au lait de chèvre dont le cahier des charges comporte un critère sur la race des animaux.

\begin{tabular}{|c|c|c|l|}
\hline Région & AOC & $\begin{array}{c}\text { Production } \\
\text { 2004 } \\
\text { tonnes) }\end{array}$ & \multicolumn{1}{c|}{ Race(s) associée(s) } \\
\hline Centre & Valençay & 348 & $\begin{array}{l}\text { Alpine } \\
\text { Saanen }\end{array}$ \\
\hline Périgord & Rocamadour & 984 & $\begin{array}{l}\text { Alpine } \\
\text { Saanen }\end{array}$ \\
\hline Massif Central & Picodon & 577 & $\begin{array}{l}\text { Alpine } \\
\text { Saanen } \\
\text { Souches locales }\end{array}$ \\
\hline \multirow{2}{*}{ Alpes } & Pélardon & 213 & $\begin{array}{l}\text { Alpine } \\
\text { Saanen } \\
\text { Rove }\end{array}$ \\
\cline { 2 - 5 } & Chevrotin & 91 & Alpine (à 80\% minimum dans le troupeau) \\
\cline { 2 - 5 } & Banon & 56 & $\begin{array}{l}\text { Commune Provençale } \\
\text { Rove } \\
\text { Alpine }\end{array}$ \\
\hline
\end{tabular}

\section{Source : INAO}

NB. Hormis dans le cas du chevrotin, le cahier des charges de ces AOC admet également les animaux issus de croisement entre les races indiquées.

\section{3 / Les fromages AOC de bre-}

a) Bilan global

L'ensemble des 3 fromages de brebis C (tableau 3) ainsi que 6 fromages portent une obligation sur la race des animaux dans leur règlement technique. La production de fromages de avec critere racial representait caprine. Ces AOC avec critère racial se situent en majorité, mais pas exclusivement, au Sud d'une ligne Bordeaux-

\section{b) Analyse par espèce et par région}

Dans le cas des fromages au lait de brebis, la liaison région-AOC-race est bassins laitiers qui existent en France ne compte qu'une seule $\mathrm{AOC}$ et une race donnée n'est associée qu'à une seule AOC également. Seul l'OssauIraty admet plus d'une race dans son 
Figure 4. Localisation des AOC fromagères ovines et caprines.

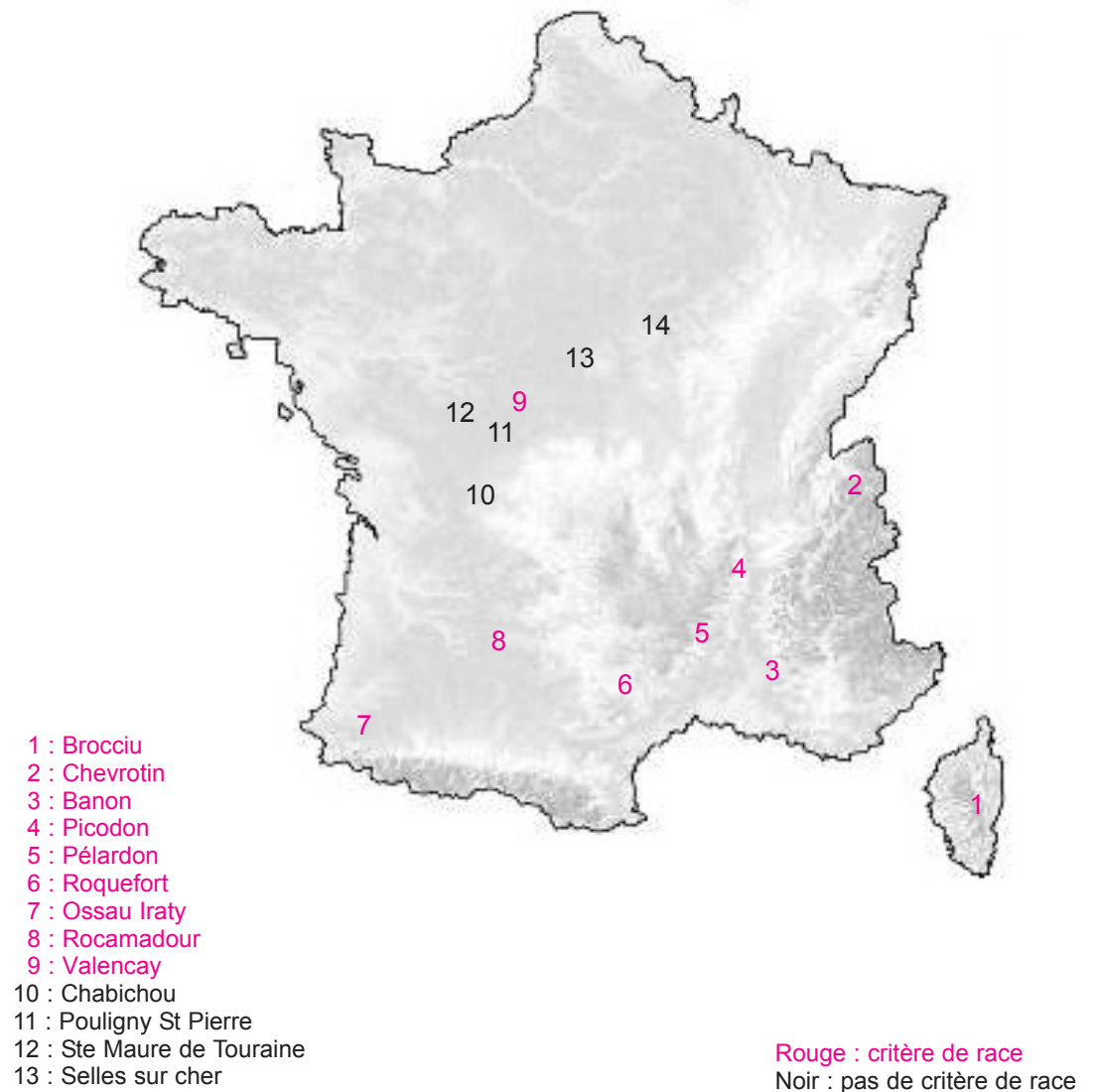

\section{4 / Les autres produits ani- maux sous AOC}

D'autres types de produits d'origine animale bénéficient d'une AOC (figure 5). Les produits laitiers autres que des fromages (beurre et crème) ne comportent aucune contrainte quant à la race des animaux dans leur cahier des charges. Les produits carnés comportent généralement une contrainte raciale, à l'exception du Fin Gras du Mézenc qui met en avant un mode spécifique d'engraissement et admet une liste «positive» de races et de croisements, et de la dinde de Bresse, qui impose un phénotype coloré et non pas un type racial. Les 4 AOC carnées avec contrainte raciale (tableau 5) présentent une forte imbrication avec leurs races «support». Dans deux cas, le taureau de Camargue et le bœuf Maine-Anjou, le choix du nom de la race pour désigner le produit a entraîné le changement du nom de la race, la législation excluant que le nom d'une AOC puisse être partagé (l'ancienne race Camargue devenant «raço di biou», et l'ancienne race Maine Anjou devenant «Rouge des Prés»). cahier des charges et, pour ces 3 fromages au lait de brebis, les races associées ont toutes leur berceau de race à l'intérieur de la zone AOC. La logique territoriale est donc très affirmée dans le cas des fromages AOC au lait de brebis.

Le cas des fromages de chèvre est particulier dans la mesure où deux races seulement, l'Alpine et la Saanen, représentent la quasi-totalité du cheptel français (RGA 2000). Ainsi, spécifier dans un cahier des charges l'une ou l'autre de ces races, et a fortiori les deux comme c'est le cas pour 4 des 6 AOC concernées, n'est pas susceptible de contribuer sensiblement à la typicité des produits. Deux races locales reconnues, la Rove et la Commune Provençale, sont impliquées dans le cahier des charges de deux AOC des Alpes du Sud. La race caprine Corse, reconnue également, est exigée pour l'élaboration du Brocciu sous AOC. Par ailleurs, une AOC fait référence à la notion, vague et à la valeur juridique discutable, de «souche locale». Ainsi, du fait d'une extrême simplification du «paysage racial» de l'espèce caprine en France, la logique territoriale de la liaison entre races animales et fromages AOC de chèvre semble moins poussée que dans le cas des autres fromages AOC.
Figure 5. Localisation des AOC d'autres produits.

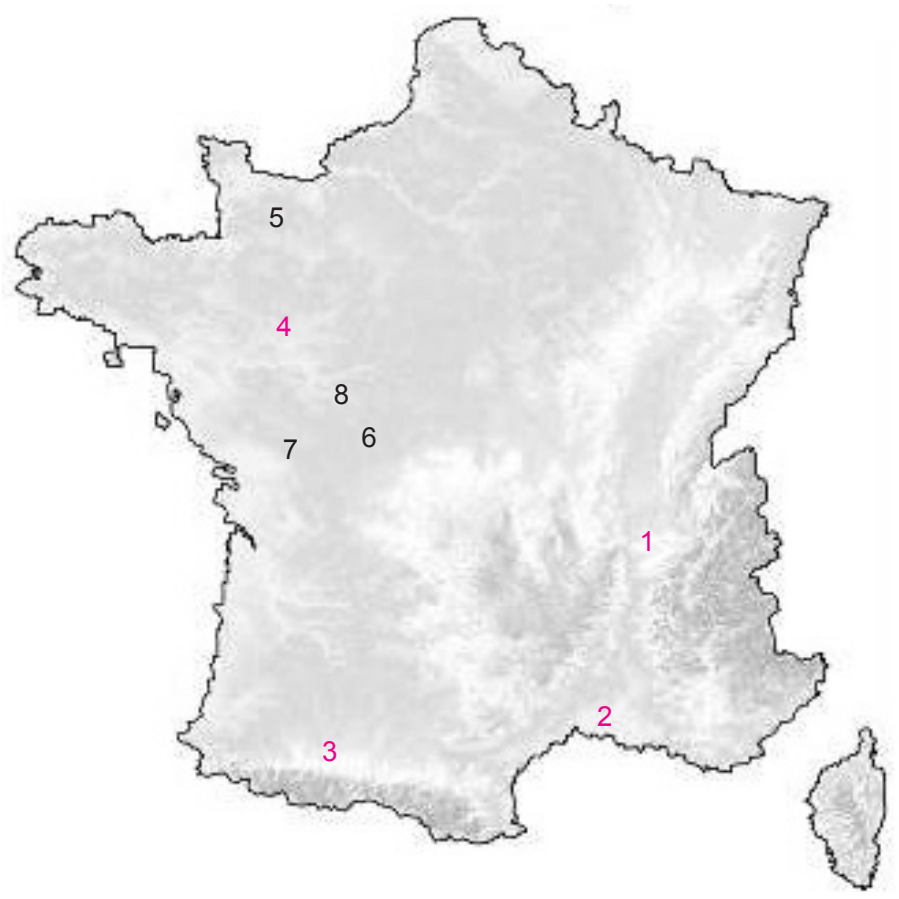

- Volaille de Bresse

: Taureau de Camargue

3 : Mouton Barrèges Gavernie

4 : Boeuf Maine Anjou

5 : Beurre et crème d'Isigny

6 : Beurre Charente Poitou

$7:$ Beurre des Charentes

8 : Beurre des deux Sèvres Noir : pas de critère de race 
Tableau 5. Produits carnés sous AOC dont le cahier des charges comporte un critère sur la race des animaux.

\begin{tabular}{|c|c|c|c|l|}
\hline Espèce & Région & AOC & $\begin{array}{c}\text { Production } \\
\mathbf{2 0 0 4} \\
\text { (tonnes }\end{array}$ & Race(s) associée(s) \\
\hline Bovins & Pays de Loire & Bœuf Maine-Anjou & n.d. & Rouge des Prés \\
\cline { 2 - 5 } & Provence & Taureau de Camargue & 310 & $\begin{array}{l}\text { Raço di Biou } \\
\text { Brava }\end{array}$ \\
\hline Ovins & $\begin{array}{c}\text { Hautes- } \\
\text { Pyrénées }\end{array}$ & $\begin{array}{c}\text { Mouton de } \\
\text { Barèges-Gavarnie }\end{array}$ & 18 & Barégeoise \\
\hline Poule & Bresse & Volaille de Bresse & 932 & Bresse (variété blanche) \\
\hline
\end{tabular}

Source : INAO.

* Pour les volailles, milliers de têtes ; n.d. = non déterminé.

\section{3 / La mobilisation des races animales par les AOC : bilan quantifié dans le cas des fromages $\mathrm{AOC}$ au lait de vache ou de brebis}

Combien d'animaux et quelle proportion d'animaux des races concernées sont mobilisés par des productions sous AOC ? Quel degré de dépendance existe-t-il entre une race et une ou des productions AOC ? Ces questions ne seront traitées ici que pour le cas des AOC fromagères au lait de vache ou au lait de brebis : outre que ces dernières représentent, avec une grande diversité de situations, la grande majorité des AOC concernées, les informations nécessaires se sont révélées peu aisées à recueillir dans le cas des produits carnés ainsi que dans le cas des fromages de chèvre.

\section{1 / La démarche mise en œuvre}

L'approche a consisté à estimer le nombre d'animaux des différentes races mobilisés par chaque AOC en fonction (i) des volumes annuels de production, (ii) de paramètres techniques de fabrication, ( $i i i)$ des contributions relatives estimées des différentes races à cette production et (iv) des données connues de productivité des cheptels. Nous avons considéré les chiffres de production de l'année 2004, présentés dans les tableaux 1 et 2 , ce qui a abouti à une «photographie» relative à cette année-là, qu'il conviendrait de
Tableau 6. Effectifs totaux et résultats de contrôle laitier des races bovines et des races ovines françaises associées, réglementairement ou non, à des fromages AOC.

\begin{tabular}{|c|c|c|c|c|}
\hline \multirow[b]{2}{*}{ Espèce } & \multirow[b]{2}{*}{ Race } & \multirow{2}{*}{$\begin{array}{l}\text { Nombre total } \\
\text { de femelles } \\
\text { reproductrices } \\
\text { en } 2000\end{array}$} & \multicolumn{2}{|c|}{$\begin{array}{c}\text { Femelles au contrôle laitier } \\
\text { en } 2004^{* *}\end{array}$} \\
\hline & & & Effectif & $\begin{array}{l}\text { Production } \\
\text { (kg lait par } \\
\text { lactation) }\end{array}$ \\
\hline \multirow{12}{*}{ Bovine } & Prim'Holstein & 2845577 & 1898150 & 8134 \\
\hline & Montbéliarde & 685043 & 381440 & 6267 \\
\hline & Normande & 577071 & 276457 & 5672 \\
\hline & Abondance & 54775 & 19896 & 5089 \\
\hline & Simmental Française & 27658 & 13767 & 5351 \\
\hline & Brune & 23942 & 16441 & 6601 \\
\hline & Tarentaise & 13488 & 7727 & 4101 \\
\hline & Bleue du Nord & 4533 & 763 & 4678 \\
\hline & Salers-lait* & 4000 & 1939 & 2425 \\
\hline & Vosgienne & 3986 & 1189 & 3676 \\
\hline & Rouge Flamande & 2394 & 863 & 5481 \\
\hline & Villard-de-Lans & 292 & 44 & 2387 \\
\hline \multirow{5}{*}{ Ovine } & Lacaune-lait* & 780000 & 177000 & 283 \\
\hline & Manech tête rousse & 277247 & 68000 & 166 \\
\hline & Manech tête noire & 120820 & 18000 & 126 \\
\hline & Basco-Béarnaise & 84962 & 18000 & 144 \\
\hline & Corse & 92215 & 20000 & 133 \\
\hline
\end{tabular}

Sources : Recensement Général Agricole ; France Contrôle Laitier ; Comité National de la Brebis Laitière.

* Le RGA ne distingue pas les rameaux laitier et allaitant de la race bovine Salers ni de la race ovine Lacaune : les effectifs totaux mentionnés ici constituent une estimation.

** Pour les brebis, contrôle laitier officiel (CLO). moduler en fonction des évolutions ultérieures.

Pour chaque fromage, un «coefficient de transformation», défini comme le nombre de $\mathrm{kg}$ de lait nécessaire à la fabrication d'un $\mathrm{kg}$ de fromage affiné (c'est-à-dire l'inverse d'un rendement fromager), a été estimé par recoupement de différentes sources : INAO, cité par Verrier et Bouffartigue 1993, Mietton et al 1994, Spinnler 2005, communication personnelle. Pour chaque fromage, on en a déduit la quantité totale de lait nécessaire à la production.

La quantité de lait totale pour une AOC donnée a ensuite été répartie entre races. A ce stade, deux situations doivent être distinguées. Pour les AOC dont le cahier des charges inclut un critère racial, nous avons directement utilisé les informations obtenues des syndicats de défense concernés (tableaux 1 et 2), jugées suffisamment fiables à ce stade (cf. plus haut). Les syndicats des AOC sans liaison réglementaire avec une race (seules les AOC au lait de vache sont concernées ici), en revanche, ont majoritairement reconnu lors de nos enquêtes ne pas disposer d'informations précises sur la composition «raciale» des laits servant aux fabrications. Dans ce cas, nous avons fait une tentative d'estimation des contributions des différentes races présentes sur la zone, à partir des statistiques du RGA de 2000 pour le ou les départements concernés. Cependant, les contours des zones AOC coïncident rarement avec ceux des départements et le bassin de collecte pour l'AOC peut avoir une structure du cheptel sensiblement différente de celle de l'ensemble du département : l'estimation obtenue ainsi est très certainement approximative. Pour toutes ces raisons, les résultats seront présentés ici de façon détaillée pour les AOC à critère racial et leurs races associées, mais seules les tendances globales seront présentées pour les autres $\mathrm{AOC}$ et les autres races.

Le passage à des nombres de femelles traites s'est enfin effectué en divisant la quantité de lait pour une $\mathrm{AOC}$ donnée en provenance d'une race donnée par la moyenne nationale de production au contrôle laitier de la race considérée, pour l'année 2004 (tableau 6). Les moyennes de contrôle laitier sont en effet, à l'échelle d'une race, les statistiques les plus fiables dont on dispose. Dans le cas des bovins, les chiffres sont fournis tous protocoles de contrôle confondus. Dans 
le cas des ovins, nous avons retenu les chiffres du Contrôle Laitier Officiel (CLO). Le choix de la moyenne nationale est discutable dans le cas des races dont la répartition ne se limite pas aux zones AOC considérées ou à des zones comparables. Cependant, les statistiques raciales de contrôle laitier les plus détaillées se situant à l'échelle des départements et, comme dit plus haut, les zones AOC ne correspondant pas strictement à des départements, seule la moyenne nationale était accessible dans cette approche.

Les effectifs obtenus sont donc des «équivalents vaches au contrôle laitier» (Eq.VCL) ou des «équivalents brebis au contrôle laitier officiel» (Eq.BCLO) nécessaires pour la production des AOC considérées. Certes, le lait des vaches ou des brebis d'un même troupeau ou d'un même bassin de collecte n'a généralement pas une destination unique, si bien que le nombre total de femelles d'une race dont la production laitière participe à une $\mathrm{AOC}$ donnée est supérieur à ce nombre équivalent. Mais ces effectifs «équivalents» traduisent bien les contributions respectives des différentes races, surtout si on les ramène aux effectifs totaux de femelles reproductrices, tels que consignés au tableau 6.

Malgré toutes les limites évoquées cidessus, la démarche employée permet d'obtenir, à partir d'informations relativement simples à collecter, une image suffisamment précise pour le phénomène que l'on cherche à cerner. Une appréciation plus précise nécessiterait des investigations sur le terrain dont la lourdeur et le coût seraient disproportionnés en regard du niveau d'analyse où l'on se situe.

\section{2 / La mobilisation des races bovines par des AOC fromagè- res}

\section{a) Bilan global}

Les résultats de l'analyse décrite cidessus, appliquée aux fromages au lait de vache avec critère racial, sont donnés au tableau 7. Un total d'environ 145400 Eq.VCL est mobilisé pour ces seules AOC ayant institué un lien réglementaire avec des races bovines. On peut estimer par ailleurs, toujours selon la méthode décrite ci-dessus, qu'environ 111200 Eq.VCL sont mobilisées pour la production des autres AOC. Ce sont donc près de $257000 \mathrm{Eq}$.VCL qui sont mobilisées par les fromages AOC au lait de vache, soit $6,5 \%$ du cheptel laitier en 2004. L'analyse qui suit porte sur la place de différentes races ou de différents groupes de races bovines.

\section{b) La prépondérance de la Montbé- liarde}

Avec les 2/3 de l'ensemble des Eq.VCL des différentes races, la Montbéliarde apparaît, de loin, comme la plus importante contributrice aux productions $\mathrm{AOC}$ à critère racial, comme le laissait pressentir sa quasiomniprésence dans les cahiers des charges (tableau 2). Dans la seule région Franche Comté, ce sont environ $95000 \mathrm{Eq}$. VCL qui sont mobilisées par les AOC, soit la moitié du cheptel régional de Montbéliardes. Dans les
Alpes du Nord, environ 14000 Eq.VCL Montbéliardes sont mobilisées par des AOC. La mobilisation globale de la Montbéliarde est évidemment plus importante encore car cette race est aujourd'hui implantée sur tout le territoire national. Notamment, la race Montbéliarde représente presque la moitié des vaches traites dans les départements laitiers du Massif Central, ces derniers constituant une importante région de production d'AOC sans critère racial (Cantal, Saint-Nectaire, Fourme d'Ambert, etc.). Selon la méthode décrite en 3.1, cette production représente en 2004 une production totale de lait de 466000 tonnes et la part revenant à la Montbéliarde correspond à environ 35000 Eq.VCL. La Montbéliarde est également présente, mais dans une moindre mesure, dans les AOC sans critère racial de l'Est de la France (Munster, Brie de Meaux, etc.). En définitive, on peut estimer qu'un total d'environ 147000 Eq.VCL de race Montbéliarde est mobilisé par des fromages AOC, toutes catégories confondues. Cela représente au minimum $21,5 \%$ de l'effectif de la race (tableau 6) - au minimum - car notre estimation se base sur des vaches contrôlées dont on sait qu'elles sont plus productives que la moyenne générale de la race.

c) Le cas des deux autres races «nationales»: ubiquité de la Prim'Holstein et régionalisation de la Normande

Le statut de la Prim'Holstein vis-àvis des AOC est singulier : exclue de

Tableau 7. Estimation de la quantité de lait nécessaire à la fabrication des fromages AOC au lait de vache dont le cahier des charges comporte un critère sur la race des animaux et du nombre de vaches mobilisées par ces AOC (en équivalent nombre de vaches contrôlées, Eq.VCL).

\begin{tabular}{|c|c|c|c|c|c|c|c|c|c|}
\hline \multirow{2}{*}{$\mathrm{AOC}$} & \multirow{2}{*}{$\begin{array}{c}\text { Coefficient } \\
\text { (kg lait / } \\
\text { kg fromage) }\end{array}$} & \multirow{2}{*}{$\begin{array}{l}\text { Quantité de } \\
\text { lait en } 2004 \\
\text { (tonnes) }\end{array}$} & \multicolumn{7}{|c|}{ Equivalent nombre de vaches au contrôle laitier } \\
\hline & & & MO & $A B$ & TA & SF & BR & VL & Total \\
\hline Comté & 12,0 & 522660 & 79229 & & & 4884 & & & 84113 \\
\hline Reblochon & 8,9 & 148069 & 11577 & 14257 & 722 & & & & 26556 \\
\hline Morbier & 9,8 & 63288 & 9594 & & & 591 & & & 10185 \\
\hline Beaufort & 12,2 & 53802 & & 5286 & 6560 & & & & 11846 \\
\hline Mont d'Or & 8,5 & 31654 & 5000 & & & 59 & & & 5060 \\
\hline Abondance & 11,4 & 17203 & 1757 & 1183 & 42 & & & & 2982 \\
\hline Laguiole* & 10,0 & 7810 & & & & 1460 & & & 1460 \\
\hline Epoisses & 8,5 & 7455 & 559 & & & 237 & 407 & & 1202 \\
\hline Tome des Bauges & 9,8 & 5821 & 464 & 343 & 284 & & & & 1091 \\
\hline Bleu de Gex & 8,1 & 4180 & 660 & & & 8 & & & 668 \\
\hline Bleu du Vercors & 8,7 & 1470 & 188 & 43 & & & & 31 & 262 \\
\hline Total & - & 863412 & 109028 & 21113 & 7608 & 7239 & 407 & 31 & 145424 \\
\hline
\end{tabular}

* Pour le Laguiole, la contribution du noyau Aubrac laitier a été négligée (cf. Tableau 2)

$\mathrm{MO}=$ Montbéliarde, $\mathrm{AB}=$ Abondance, $\mathrm{TA}=$ Tarentaise, SF = Simmental Française, $\mathrm{BR}=\mathrm{Brune}, \mathrm{VL}=\mathrm{Villard}-\mathrm{de}-\mathrm{Lans}$. 
toutes les AOC qui intègrent un critère sur la race des animaux, elle contribue de façon substantielle à toutes les autres AOC du fait de son importance numérique (deux vaches laitières sur trois, tableau 6) et de sa présence sur quasiment tout le territoire. D'après les statistiques du RGA de 2000, la Prim'Holstein représente environ $40 \%$ du cheptel des zones AOC du Massif Central, $50 \%$ pour les AOC normandes (Camembert de Normandie, Pontl'Evêque, etc.), 75 \% pour les AOC du "grand Est» hors Franche-Comté (Munster $=75 \%$, Brie de Meaux > $75 \%$, Langres $<75 \%$, etc.) et plus de $95 \%$ pour le Nord-Pas-de-Calais (Maroilles). Au total, nos calculs conduisent à une estimation de 52000 Eq.VCL de race Prim'Holstein mobilisées par des AOC. Ce chiffre ne représente «que» le tiers de celui relatif à la Montbéliarde mais situe la Prim'Holstein au $2^{\text {ème }}$ rang des races laitières pour le nombre d'Eq.VCL mobilisées, réglementairement ou non, par des AOC. En proportion, en revanche, les AOC ont un impact mineur pour la Prim'Holstein, puisqu'elles mobilisent $1,8 \%$ du cheptel de la race.

Les effectifs nationaux de la race Normande sont encore importants (tableau 6) mais son aire d'extension s'est fortement réduite ces dernières décennies pour aujourd'hui se limiter quasiment à la région qui lui a donné son nom. Ainsi, la Normande n'est mobilisée, pour l'essentiel, que par les AOC normandes. Le cahier des charges de ces dernières n'incluant pas de critère sur la race des animaux ${ }^{11}$, la Normande doit ici «partager le terrain» avec la Prim'Holstein (cf. plus haut), les autres races ayant une présence marginale. En définitive, nous estimons qu'environ 12400 Eq.VCL de race normande sont mobilisées par des fromages AOC, soit seulement 2,1 \% du cheptel de la race.

d) Le lien étroit entre les races Abondance et Tarentaise et les AOC de leur région d'origine

Les deux races locales des Alpes du Nord, Abondance et Tarentaise, présentent des situations sensiblement différentes de celles des races dites nationales. Leurs nombres d'Eq.VCL mobilisées par des $\mathrm{AOC}$ à critère racial

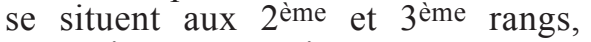
respectivement, mais sont nettement plus faibles que celui de la Montbéliarde (tableau 7). L'aire d'ex- tension de ces deux races est aujourd'hui centrée sur leur berceau d'origine: la Tarentaise n'est plus présente dans d'autres bassins laitiers que celui des Alpes du Nord et l'Abondance ne représente plus que 2 à $3 \%$ du cheptel des départements laitiers du Massif Central. Ainsi, le nombre total d'Eq.VCL Tarentaises mobilisées par des AOC est restreint à celui relatif aux AOC avec critère racial. On peut estimer qu'environ $2300 \mathrm{Eq}$.VCL de race Abondance sont mobilisées par les AOC du Massif Central, soit un total de 23400 Eq.VCL mobilisées par tout type d'AOC. Ces deux races locales présentent en revanche de très fortes proportions de vaches mobilisées par des AOC, avec au minimum $55 \%$ des vaches Tarentaises et $43 \%$ des vaches Abondance.

e) Les cas particuliers de la Simmental Française et de la Salers

La race Simmental Française se caractérise par une valeur d'Eq.VCL mobilisées par des $\mathrm{AOC}$ à critère racial du même ordre de grandeur que celle de la Tarentaise (tableau 7). Cette valeur est liée, d'une part, à sa présence en Franche-Comté, grande région de production de fromages AOC, et, d'autre part, au développement d'un noyau Simmental autour de la production du Laguiole (cf. plus haut). La Simmental Française étant également présente dans le grand Est et, dans une moindre mesure, dans le Massif Central hors zone du Laguiole, elle se trouve encore mobilisée par d'autres AOC pour environ 1300 Eq.VCL. L'ensemble représente $8500 \mathrm{Eq}$.VCL mobilisées, soit au minimum $31 \%$ du cheptel. Race d'origine internationale, présente en faible nombre dans différentes régions et véhiculant donc une image «terroir» moins affirmée que des races locales, la Simmental Française n'en demeure pas moins une race fortement mobilisée par les AOC.

La Salers, quant à elle, est exploitée en tant que vache traite dans les départements du Cantal et du Puy-de-Dôme. Nous estimons qu'environ 3900 Eq.VCL sont mobilisées par les AOC Cantal, Saint-Nectaire, Salers et Fourme d'Ambert. Cette estimation souffre sans doute d'un manque de précision mais il est vraisemblable que la quasi-totalité des vaches Salers encore traites le soient en vue d'une production sous AOC.

\section{f) Les autres races}

Les autres races réglementairement associées à des AOC, Brune et Villardde-Lans, représentent de faibles valeurs d'Eq.VCL mobilisées mais dans le cas de la Villard-de-Lans, race en conservation, ce sont environ $10 \%$ des vaches qui sont mobilisées (tableau 7). Les AOC sans critère racial mobilisent vraisemblablement en plus quelques centaines d'Eq.VCL Brunes (Cantal, Langres,...) et Vosgiennes (Munster) et quelques dizaines d'Eq. VCL Bleues du Nord et Rouges Flamandes (Maroilles).

\section{3 / La mobilisation des races ovines}

Le tableau 8 fournit les résultats de l'analyse quantifiée de la liaison raceproduit dans le cas des fromages AOC au lait de brebis. Le Brocciu mis à part, les AOC au lait de brebis mobilisent un total de presque 400 milliers d'Eq.BCLO, soit environ un tiers du cheptel. Du fait de l'exclusivité de la liaison (une race n'est associée qu'à une seule $\mathrm{AOC}$ ) et du nombre restreint d'AOC concernées, la situation est beaucoup plus simple que dans le cas des fromages au lait de vache.

Le nombre de brebis mobilisées pour la production de Roquefort paraît ne représenter que $37 \%$ du cheptel Lacaune-lait et ceci doit s'interpréter de plusieurs façons. Tout d'abord, il s'agit d'équivalent brebis au contrôle laitier officiel et le nombre de brebis qui y sont soumises ne représente environ qu'un quart du total de la population : le décalage de production avec la brebis «moyenne» est donc relativement marqué et le nombre d'équivalent brebis «moyennes» serait sensiblement plus élevé (de l'ordre de 350 000). Par ailleurs, du fait de la saturation du marché, les transformateurs de la zone de Roquefort ont développé un certain nombre de produits de diversification. Enfin, la race Lacaune s'est implantée dans toutes les régions où l'élevage de brebis laitières s'est récemment développé.

Le nombre total d'Eq.BCLO mobilisées par 1'Ossau-Iraty représente entre 20 et $25 \%$ du cheptel de chacune des trois races concernées. Ainsi, le développement des races locales pyrénéennes ne peut pas s'appuyer uniquement sur cette AOC, bien que le cahier des charges de cette dernière en stipule l'usage exclusif.

11 Des modifications de décrets sont en cours pour ces AOC Normandes (Neufchâtel, Livarot, Pont l'Evêque et Camembert), avec entre autres l'introduction d'un critère sur la race Normande, avec des degrés d'implication dans la production assez variables. 


\section{4 / Questions et enjeux autour de la liaison entre AOC et races animales}

L'importance actuelle de la prise en compte d'un critère racial dans le cahier des charges des produits animaux sous AOC et son accroissement dans le temps soulèvent plusieurs types de question : quels apports réciproques, quel lien entre la race et le lieu, quels enjeux pour la gestion génétique des races concernées ? Ces questions se déclinent différemment selon qu'elles concernent des races locales ou des races de plus large extension.

\section{1 / Apports réciproques entre $\mathrm{AOC}$ et races animales}

a) La valorisation économique liée aux $A O C$

La production sous AOC s'accompagne généralement de la recherche de prix élevés payés aux producteurs pour leurs produits (la «matière première»). Le signe $\mathrm{AOC}$ ne suffit cependant pas à lui seul à obtenir ces prix élevés. L'organisation des éleveurs, leur degré de contrôle sur les filières et donc leur capacité à avoir un poids décisionnel sur la fixation des prix, d'une part, et le degré d'exigence du cahier des charges de l'AOC, d'autre part, se révèlent également déterminants. Par exemple, en ce qui concerne les fromages AOC au lait de vache, en dehors des productions fermières, on peut schématiquement opposer, d'un côté, les AOC du Massif Central - hors Laguiole - et, de l'autre, les AOC de Franche-Comté et des Alpes du Nord ainsi que le Laguiole (Ricard 1994, Chatellier et Delattre 2003). Les AOC du premier groupe ont un cahier des charges peu exigeant, qu'il s'agisse du type d'animaux, de leur alimentation, du type de lait (cru ou pasteurisé) ou des durées d'affinage. Elles sont fabriquées dans des ateliers de groupes coopératifs ou privés d'envergure nationale et ne constituent qu'un élément parmi leur gamme de produits. Dans ces conditions, le prix du lait payé aux éleveurs est équivalent à la moyenne nationale. Les AOC du second groupe ont un cahier des charges exigeant, voire extrêmement exigeant, sont produites par des coopératives ayant un rayon d'action limité (exemple des fruitières villageoises de Franche-Comté) dont elles constituent l'essentiel de la production : ici, le prix du lait payé aux éleveurs se situe aux niveaux les plus élevés. Par exemple, les fromages AOC des Alpes du Nord permettent aux éleveurs concernés d'obtenir un prix du lait supérieur de 20 à $40 \%$ au prix du lait destiné à des productions non-AOC dans la même région et de 35 à $60 \%$ au-dessus de la moyenne nationale (Ricard 1994, Verrier 1995, Chatellier et Delattre 2003). Dans un autre secteur, la volaille de Bresse est le fait de producteurs très organisés au sein d'une filière bien encadrée et se caractérise par un cahier des charges très exigeant : en se fondant sur le poulet entier prêt à cuire, le prix au kg payé aux éleveurs est supérieur au prix du $\mathrm{kg}$ sur le marché de Rungis, d'environ $50 \%$ par rapport au poulet label et $150 \%$ par rapport au poulet standard (Verrier et al 2005).

\section{b) Les atouts des races pour les $A O C$}

La prescription ou non de l'usage de certaines races animales est un des éléments du degré d'exigence du cahier des charges d'une AOC. La mobilisation d'une ou de quelques races peut ainsi contribuer à tirer les prix vers le haut. L'apport d'une race à une AOC peut être de deux natures : d'une part, des caractéristiques zootechniques moyennes qu'il est possible d'établir objectivement et qui peuvent constituer des atouts pour la production et, d'autre part, une image. Par exemple, les deux races bovines locales des Alpes du Nord, mobilisées par chacune des AOC de la région, présentent des traits d'adaptation au milieu montagnard favorables au maintien d'un système d'élevage qui implique la transhumance et l'exploitation des alpages (Anonyme 1972, D'Hour et al 1994). Le lait de ces races présente de bonnes aptitudes fromagères, qu'il s'agisse de la richesse en matières protéiques (résultats de contrôle laitier) que de caractéristiques plus fines (Macheboeuf et al 1993). Ces deux races apportent également une indéniable image «montagne» qui souligne le lien au terroir revendiqué par les $\mathrm{AOC}$ et une image «naturelle» favorable à la promotion de ces fromages. D'une manière générale, l'image des races qui ne sont perçues ni comme «dominantes» ni liées aux productions «standard» est utilisée pour contribuer à démarquer les produits. A titre d'exemple, les robes colorées de certaines races bovines, associées à des paysages herbagers ou de montagne, font contraste avec la robe pie noire associée aux systèmes intensifs de plaine.

\section{c) De l'intérêt d'une valorisation des} races locales

La liaison réglementaire entre $\mathrm{AOC}$ et races est également bénéfique pour ces dernières, au moins sur le plan démographique, car une telle liaison leur assure un créneau d'utilisation qui peut correspondre à des effectifs d'animaux conséquents (tableaux 7 et 8). Cet apport est surtout sensible pour les races locales, dont les effectifs ont généralement diminué depuis le milieu du XX ${ }^{\text {ème }}$ siècle. Par exemple, dans les Alpes du Nord, la prise en compte d'un critère racial dans le cahier des charges des $\mathrm{AOC}$, couplée à un effort d'aménagement de l'accès aux alpages et d'amélioration des conditions de vie et de travail en alpage, a sans aucun doute contribué à la stabilisation des effectifs de la race Tarentaise et à la reprise de ceux de la race Abondance observées au cours de la dernière décennie (Verrier et al 2005). De façon plus spectaculaire encore, c'est indéniable-

Tableau 8. Estimation de la quantité de lait nécessaire à la fabrication des fromages AOC au lait de brebis dont le cahier des charges comporte un critère sur la race des animaux et du nombre de brebis mobilisées par ces AOC (en équivalent nombre de brebis au contrôle officiel, Eq.BCLO).

\begin{tabular}{|c|c|c|c|c|c|c|c|}
\hline \multirow[t]{2}{*}{$\mathrm{AOC}^{*}$} & \multirow{2}{*}{$\begin{array}{c}\text { Coefficient } \\
\text { (kg lait / kg } \\
\text { fromage) }\end{array}$} & \multirow{2}{*}{$\begin{array}{l}\text { Quantité de lait } \\
\text { en } 2004 \\
\text { (tonnes) }\end{array}$} & \multicolumn{5}{|c|}{$\begin{array}{l}\text { Equivalent nombre de brebis } \\
\text { au contrôle laitier officiel }\end{array}$} \\
\hline & & & LA & MTR & MTN & BB & Total \\
\hline Roquefort & 4,3 & 80973 & 286125 & & & & 286125 \\
\hline Ossau-Iraty & 4,8 & 1690 & & 56217 & 30647 & 20112 & 106976 \\
\hline Total & - & 82663 & & & & & 393100 \\
\hline
\end{tabular}

* Le Brocciu, qui mobilise la race ovine Corse, n'est pas considéré ici pour cause de non détermination du coefficient de transformation et d'impossibilité de dissocier les apports respectifs des laits de brebis et de chèvre.

LA = Lacaune, MTR = Manech tête rousse, MTN = Manech tête noire, BB = Basco-Béarnaise. 
ment le développement de l'AOC «volaille de Bresse» à partir des années 60 qui a permis à la race du même nom, autrefois maintenue uniquement par des éleveurs «collectionneurs», de se réinsérer dans une filière économique (Verrier et al 2005).

Plus généralement, la valorisation des races locales constitue une garantie de leur maintien dans un tissu économique et social vivant (Audiot et al 1993, 2005). Cela prend des formes variées : signes d'origine ou de qualité, circuits courts, etc. En dehors des races associées à des $\mathrm{AOC}$ citées dans cet article, on peut évoquer plusieurs exemples de races locales autrefois menacées et ayant réussi à se maintenir ou à se développer grâce, entre autres, à une bonne valorisation : les races porcines Basque (Arrayet et al 2003), Gasconne (Audiot et al 2003 ) et Corse (Audiot et al 2005) ; certaines races anciennes de poulet (TixierBoichard et al 2006) ; la race bovine Parthenaise (Giraudeau 1990) ; la race bovine Bretonne Pie Noire (Quéméré et al 1999) ; etc.

\section{2 / De la réalité du lien au lieu}

La politique d'introduction de critères raciaux dans les cahiers des charges des AOC a été menée dans l'intention de renforcer le lien au terroir, notamment en vue de l'harmonisation européenne. La race locale a donc représenté un argument potentiel en faveur de la défense du lien au lieu. De ce point de vue, l'analyse présentée dans cet article indique clairement que la réalité est multiforme.

a) La race, élément constitutif de l'origine?

Certaines races, dans certaines AOC, jouent un rôle effectif dans l'établissement du lien au lieu, c'est-àdire qu'à la fois elles donnent une image «terroir» au produit et elles contribuent substantiellement à l'originalité des productions. C'est le cas de races locales exploitant un milieu difficile, notamment en montagne : races bovines Abondance et Tarentaise, races ovines pyrénéennes et corse. C'est aussi le cas de races ayant une longue histoire dans une région donnée, comme la race bovine Montbéliarde, la race ovine Lacaune ou la race avicole de Bresse.

Le cas de la Montbéliarde qui vient d'être cité illustre bien la complexité de l'établissement du lien au lieu. Si cette race est en effet emblématique de l'élevage en Franche-Comté et de ses produits, il n'en est pas de même dans d'autres régions où elle est également associée à des AOC. De façon plus nette encore, l'usage des races caprines Alpine ou Saanen ne peut pas être motivé par l'expression d'un lien à une région ou à un système d'élevage, dans la mesure où ces deux races sont présentes sur tout le territoire national et dans tout type de système d'élevage. La Simmental Française, quant à elle, est mobilisée loin de son berceau d'origine, dans des régions où elle s'est implantée grâce à ses atouts et où elle permet de maintenir certaines caractéristiques des systèmes d'élevage même après l'abandon de la race locale traditionnelle (cas de l'AOC Laguiole). Si l'on peut admettre la nécessité d'être pragmatique face à l'implantation des races dans une zone AOC au moment d'établir un critère racial dans son cahier des charges, on peut se demander de quelle antériorité une race a besoin ou de quelles aptitudes elle doit faire preuve pour assurer sa légitimité.

\section{b) La race, alibi marketing?}

Il suffit d'examiner les étiquettes de différents produits, en particulier les étiquettes de fromages, pour se convaincre que l'image d'une race locale est un élément au service de la communication envers le consommateur. De nombreux exemples d'AOC montrent que la race choisie pour communiquer est celle qui véhicule la plus forte image sans pour autant qu'elle soit la seule utilisée ni, le plus souvent, qu'elle soit la race majoritaire. En se limitant aux fromages au lait de vache (tableau 7 et $\S 3.2$ ) on peut citer la Normande pour le Camembert de Normandie, la Salers pour le SaintNectaire et le Cantal, l'Abondance pour le Reblochon et l'Abondance (fromage), ou la Tarentaise pour le Beaufort.

Il est possible d'intégrer une race locale dans le cahier des charges d'une AOC afin d'en relancer le développement tout en bénéficiant de son image (cf. cidessus). L'association de la race Aubrac avec le Laguiole et de la Villard-de-Lans avec le Bleu du Vercors ressort sans doute de ces deux motivations. Lorsque la contribution de la race en question est marginale et le demeure dans la durée, on est en droit de se demander si la recherche d'une image ne constitue pas alors l'unique motivation de la démarche.

\section{3 / Enjeux autour de la gestion des races concernées}

\section{a) Le nom, enjeu d'image}

Le nom d'une race, généralement lié à un lieu, constitue un patrimoine que le collectif doit également gérer. Jusqu'au début des années 90, un nom de lieu qui était déjà le nom d'une race animale a pu être donné à une AOC : volaille de Bresse, fromage d'Abondance, etc. Cependant, le règlement européen $\mathrm{n}^{\circ} 2081 / 92$ du 14 Juillet $1992^{12}$ interdit qu'un même nom désigne à la fois une race et un produit. Depuis cette époque, donc, des «conflits» quant à l'usage du nom se sont présentés lors de la création de nouvelles AOC. Dans plusieurs cas, le nom étant porteur d'une image forte, il est donné à l'AOC et la race doit changer de nom : ainsi, la race bovine Camargue est devenue «raço di biou», la race bovine Maine-Anjou est devenue «Rouge des prés» et, dans le cadre d'un dossier en cours, il a été décidé que la race porcine Corse devienne la race «Nustrale». Parfois, il est possible de trouver un nom d'AOC porteur d'image (mouton de BarègesGavarnie) sans que la race (ici, la race Barégeoise) change de nom. De tels compromis sont des objets qui méritent d'être étudiés afin de comprendre les mécanismes de structuration permettant d'aboutir.

b) La cohérence entre les pratiques d'élevage et les aptitudes des animaux

La construction des cahiers des charges AOC se fait sur la base de compromis, entre autres autour des conditions de production dans lequel la race est incluse. De par leur aptitude à exploiter un terroir donné, les races locales peuvent être garantes de la continuité de systèmes d'élevage en lien direct avec la culture locale. Les cas de l'Abondance et de la Tarentaise dans les Alpes du Nord (Verrier et al 2005) ou de la race Barégeoise dans les Hautes Pyrénées (Roncin 2000) sont exemplaires à cet égard. La pratique de la transhumance, spécifique à ces systèmes d'élevage en région de montagne, soumet les animaux à des contraintes particulières : variations climatiques (orages d'été, neige précoce ou tardive), topographie accidentée etc.

12 Article 3, alinéa 2 du Règlement (CEE) N 2081/92 du conseil du 14 Juillet 1992, relatif à la protection des indications géographiques et des appellations d'origine des produits agricoles et des denrées alimentaires, Journal officiel des Communautés européennes, $\mathrm{N}^{\circ}$ L 208/1. 
L'aptitude des animaux à s'adapter à ces systèmes particulièrement contraignants devient donc un critère fort dans le choix des races (cf. 4.1.b). Ces exemples montrent bien l'indispensable cohérence à maintenir entre aptitudes des animaux et pratiques d'élevage au sein d'une AOC afin d'assurer le lien au terroir et le maintien des «usages locaux, loyaux et constants». C'est bien le particularisme de chaque situation qui donne alors à l'AOC tout son sens.

Une contrainte particulièrement exigeante consiste, dans quelques AOC fromagères, à fixer une limite aux performances laitières (figures 3 et 4). Outre les répercussions possibles en matière de choix des races et de sélection de ces dernières (voir ci-après), cela s'accompagne généralement de recommandations en matière de pratiques d'élevage qui vont dans le même sens, notamment en matière d'alimentation : recours majoritaire au pâturage et aux fourrages récoltés sur place, usage de parcours, limitation de l'apport en concentrés. Se pose alors la question de la marge de manœuvre des éleveurs pour maintenir une cohérence entre choix des reproducteurs et pratiques d'élevage.

Une difficulté se retrouve aussi dans les cas des AOC où la zone de production recouvre un territoire vaste et hétérogène et permet ainsi l'expression d'une gamme diversifiée de systèmes d'élevage (dans le respect du règlement technique). On peut citer à ce propos les AOC caprines, où l'on rencontre des systèmes basés sur l'utilisation des parcours, et d'autres sur le principe du zéro pâturage. Les cas de l'Ossau Iraty, de l'Abondance et du Reblochon reposent aussi sur des situations où cohabitent des systèmes montagnards avec transhumance, et des systèmes relativement intensifiés de piémont. Quelle cohérence est permise au sein de cette diversité, et quelle homogénéité peut être recherchée dans le choix des animaux reproducteurs?

c) Multiplicité des acteurs et orientations génétiques

La mobilisation de races par des filières spécifiques, ici des filières AOC, peut entraîner une implication des acteurs de ces filières dans les collectifs de gestion de ces races. Jusqu'à la fin de l'année 2006, l'orientation génétique de chaque race et le choix de ses objectifs de sélection constituaient une des missions des Unions pour la Promotion et la sélection des Races (UPRA). Les UPRA étaient constituées de trois collèges : un collège d'éleveurs à titre individuel, un collège d'entreprises collectives de sélection (unions de coopératives d'insémination) et un collège d'organismes utilisateurs. Force est de constater que, dans les faits, la place des syndicats $\mathrm{AOC}$ dans le 3 ème collège était hétérogène d'une race à l'autre. La loi d'orientation agricole de janvier 2006, qui remplace la loi sur l'élevage de 1966 dans le cadre de laquelle ont été créées les UPRA, prévoit la création d'Organismes de Sélection qui reprendront cette mission d'orientation et au sein desquels la place de l'aval est réaffirmée.

Lorsqu'une race est fortement mobilisée par une AOC (tableaux 7 et 8), les règles du cahier des charges et les décisions prises pour son évolution peuvent avoir des conséquences directes sur les objectifs et les pratiques de sélection. Par exemple, c'est le nécessaire respect du critère de taux de gras sur sec dans l'AOC Roquefort qui a motivé la prise en compte effective du taux de matière grasse du lait en race ovine Lacaune et la mise en place d'un protocole spécifique de mesure de ce caractère au sein des élevages (Barillet 1985). De même, dans le cas de l'AOC Beaufort, certaines règles de production ont un impact direct sur la sélection des deux races autorisées : la limite actuelle de production de $5000 \mathrm{~kg}$ de lait par vache et par an à l'échelle de chaque troupeau impose un effort de sélection modéré sur la quantité de lait (ou sur les quantités de matières utiles), tout particulièrement en race Abondance dont la moyenne de contrôle laitier dépasse déjà cette limite (tableau 6) ; la fixation à $6,25 \%$ d'une limite du pourcentage de gènes Holstein chez les vaches Abondance, mesure en cours de discussion dans le syndicat, constituerait une intervention directe dans le débat qui a lieu au sein de l'UPRA Abondance au sujet d'une éventuelle élimination des gènes Holstein introduits par croisement dans le courant des années 80 et 90 .

Pour des races étroitement liées dans les faits à une AOC (races bovines Tarentaise ou Rouge des Prés, ex MaineAnjou, race ovine Lacaune, race avicole de Bresse, etc.), les décideurs au sein des UPRA ou des associations de race et ceux des syndicats AOC peuvent arriver rapidement à une convergence de vues (quand il ne s'agit pas des mêmes personnes, pour l'essentiel) : l'AOC «tire» simultanément la filière et la race, qui peuvent s'accorder sur les objectifs en ce qui concerne les aptitudes des animaux. Il en va autrement pour les races liées à des AOC mais également largement pré- sentes hors zone, comme la race bovine Montbéliarde. Dans ce cas, quel collectif pèse le plus dans les décisions : celui du berceau et des zones AOC ou celui des régions d'extension? Dans le cas de la Montbéliarde, comment fournir une gamme de taureaux d'insémination dans laquelle à la fois les éleveurs Franc-comtois encore très liés aux AOC et les éleveurs des zones d'extension trouveront des reproducteurs présentant les aptitudes génétiques qu'ils recherchent ? Quelle capacité à proposer une déclinaison interne à la race, en rajoutant des mentions à certains reproducteurs pour en spécifier les aptitudes dans l'offre raciale ? Dans ce type de situation, la qualité du dialogue entre les différents collectifs se révèle déterminante.

\section{Conclusion}

A l'instar des cépages dans le règlement technique des AOC vinicoles, la mobilisation des races par les AOC animales est aujourd'hui une réalité qui semble aller en s'accentuant. La race locale est effectivement devenue un facteur important dans l'argumentaire des demandes d'AOC. La situation des races dans ces AOC est cependant diversifiée : la majorité d'entre elles sont mobilisées à la fois pour des produits avec ou sans $\mathrm{AOC}$, quelques races sont dédiées à certaines AOC, d'autres ne sont qu'à peine sollicitées, et d'autres, enfin, ne doivent leur contribution qu'à l'absence de critères dans les règlements techniques et à leur extension à tout le territoire. Si elles contribuent parfois aux conditions d'expressions d'un terroir, ces races peuvent en revanche, n'être que de simples arguments de marketing.

A l'exception de quelques cas particuliers, les collectifs d'AOC et les collectifs de race, sans être disjoints, ne sont que faiblement imbriqués. Deux éléments méritent alors réflexion : au sein de l'AOC, y a-t-il une vision convergente de la race et de son devenir ? Et au sein du collectif utilisateur de la race, cette AOC représente-t-elle un poids dans les prises de décisions ? En parallèle, la mise en place des Organismes de Sélection prévus par la Loi d'Orientation Agricole, met à nouveau l'accent sur la place des utilisateurs dans la construction des compromis parmi les gestionnaires des races. Des configurations diverses peuvent alors apparaitre, et il est donc particulièrement important d'analyser comment les dynamiques AOC ou tout autre dynamique de valorisation, font irruption dans la gestion des ressources génétiques animales. 


\section{Références}

Anonyme, 1972. Comportement des bovins en alpage. Etude INERM, 59, 200p.

Arrayet J., Montel B., Lossouarn J., 2003. Small is beautiful... but how to remain small? A case-study about the Basque swine system and its related market chain. $6^{\text {th }}$ Int. Liv. Farming Systems Symp., Benevento, Italy, 26-29 August 2003, 7p.

Audiot A., Bougler J., Verrier E., 1993. Peutil $\mathrm{y}$ avoir conservation sans valorisation? Ethnozootechnie, 52, 19-24.

Audiot A., Roche B., Onco-Barella M., 2003. How does the development of typical products act upon animal qualification in endangered breeds? The case study of the Gascon pig. $6^{\text {th }}$ Int. Liv. Farming Systems Symp., Benevento, Italy, 26-29 August 2003, 6p.

Audiot A., Bouche R., Brives H., Casabianca F., Gaillard C., Roche B., Trift N., Steyaert P., 2005. Populations animales locales et produits de qualité : comment valoriser transforme la ressource? Les Actes du BRG, 5, 577-592.

Béranger C., Hédin L., Kerguelen M., Petit M., Teissier J.H., 1970. Productions fourragères et méthodes d'élevage. In : L'Aubrac, Etude ethnologique, linguistique, agronomique et économique d'un établissement humain, Editions du CNRS, Tome 1, 103-165.

Bérard L., Delfosse C., Marchenay P., 2004. «Les produits de terroir»: de la recherche à l'expertise. Ethnologie française, 34, 561-600.

Barillet F., 1985. Amélioration génétique de la composition du lait des brebis : l'exemple de la race Lacaune. Thèse de docteur-ingénieur, INA Paris-Grignon, 144p.

Boichard D., Maignel L., Verrier E., 1996. Analyse généalogique des races bovines laitières françaises. INRA Prod. Anim., 9, 323-335.

Casabianca F., Sylvander B., Noel Y., Béranger C., Coulon J., Roncin F., 2005. Terroir et Typicité : deux concepts clés des Appellations d'Origine Contrôlée. Essai de définitions scientifiques et opérationnelles. In : Territoires et enjeux du développement régional, Lyon, France, $18 \mathrm{p}$.
Chatellier V., Delattre F., 2003. La production laitière dans les montagnes françaises : une dynamique particulière pour les Alpes du Nord. INRA Prod. Anim., 16, 61-76.

CNPL-INAO, 2002. Compte rendu de réunion, commission «conditions de production», 10p.

D'Hour, P., Hauwuy, A., Coulon, J.B., Garel, J.P., 1994. Walking and dairy cattle performance. Ann. Zootech., 43, 369-378.

Giraudeau L., 1990. La gestion de la variabilité génétique d'une race d'effectif limité : le cas de la race bovine Parthenaise. Mémoire de fin d'études, INA Paris-Grignon, France, 116p.

Grosclaude F., Aupetit R.Y., Lefebvre J. Mériaux J.C., 1990. Essai d'analyse des relations génétiques entre les races bovines françaises à l'aide du polymorphisme biochimique. Genet. Sel. Evol., 22, 317-338.

Le Jaouen J., Dubeuf J., Rubino R., 2001. Le système de production dans le cahier des charges des fromages caprins et ovins AOC de l'Union Européenne. In: Production systems and product quality in sheep and goats. Les systèmes de production et la qualité des produits en ovins et caprins, M.-F. P. Rubino R., (Ed.), CIHEAMIAMZ, Zaragoza, Espagne, 81-92.

Macheboeuf, D., Coulon, J.B., D'Hour, P., 1993. Aptitude à la coagulation du lait de vache. Influence de la race, des variants génétiques des lactoprotéines du lait, de l'alimentation et du numéro de lactation. INRA Prod. Anim., 6, 333344.

Mattalia S., Barbat A., Danchin-Burge C., Brochard M., Le Mézec P., Minéry S., Jansen G., Van Doormaal B., Verrier E., 2006. La variabilité génétique des principales races bovines laitières françaises : quelles évolutions, quelle comparaisons internationales ? Renc. Rech. Rum., 13, 239-246.

Mietton B., Desmazeaud M., Roissart H. de, Weber M., 1994. Transformation du lait en fromage. In : Les bactéries lactiques, $H$. de Roissard (Ed), 2, Lorica, Uriage, 55-133.

Quéméré P., Bougler J., Brossard, G., Sergent J., 1999. La race bovine bretonne Pie-Noire
$(\mathrm{BPN})$ : de la sauvegarde à la relance. Renc. Rech. Rum., 6, 43-46.

Prost J., Casabianca F., Casalta E., Vallerand F., de Sainte Marie C., 1994. La certification des produits, un levier pour le développement de l'élevage. La dynamique de l'AO «Brocciu Corse». Etudes et Recherches sur les Systèmes Agraires et le Développement, 28, 143-156.

Ricard D., 1994. Les montagnes fromagères en France. CERAMAC-Editions, Université Blaise Pascal, Clermont-Ferrand, 495p.

Roncin F., 2000. Les enjeux de la conservation des races locales, position du problème pour les signes de qualité: cas des appellations d'origine. In : Races locales et biodiversité animale ; Enjeux et appétits, Actes du colloque FAIR, Rambouillet, France, 147-157.

Tixier-Boichard M., Audiot A., Bernigaud R. Rognon X., Berthouly C., Magdelaine P., Coquerelle G., Grinand R., Boulay M., Ramanantseheno D., Amigues Y., Legros H., Guintard C., Lossouarn J., Verrier E., 2006. Valorisation des races anciennes chez le poulet : facteurs sociaux, technico-économiques, génétiques et règlementaires. Les Actes du BRG, 6 , 495-520.

Verrier E., Bouffartigue B., 1993. Les AOC, élément du maintien des races régionales? L'exemple des Alpes du Nord et réflexions sur d'autres situations. Ethnozootechnie, 52, 33-42.

Verrier E., 1995. La place des races bovines Abondance et Tarentaise dans une politique d'aménagement du territoire des Alpes du Nord. II - Une dynamique nouvelle en cours. Bull. Acad. Vét. Fr., 68, 193-200.

Verrier E., Tixier-Boichard M., Bernigaud R., Naves M., 2005. Conservation and values of local livestock breeds: usefulness of niche products and/or adaptation to specific environments. Anim. Genet. Res. Inf., 36, 21-31.

Vissac B., 2001. Les vaches de la république. INRA Edition, Paris, France, 505p.

\section{Résumé}

L'intégration d'un critère racial dans le règlement technique des produits animaux sous AOC est en nette augmentation depuis les années 90. Elle concerne aujourd'hui l'intégralité des fromages AOC au lait de brebis, la majorité des AOC carnées, environ la moitié (en quantité) de la production de fromage AOC au lait de vache et le tiers de celle de fromage de chèvre AOC. Des logiques régionales apparaissent clairement, traduisant ainsi les différentes conceptions de l'AOC portés par les acteurs de ces filières. Dans le cas de la production fromagère, nous avons estimé que l'équivalent de $6,5 \%$ des vaches laitières et d'environ un tiers des brebis laitières sont mobilisées par des AOC. L'analyse détaillée selon les races révèle une grande diversité de situations. Les atouts de ce lien race/AOC sont analysés. Il apparaît que la race peut contribuer à la typicité des produits et permet ainsi d'étayer la notion d'origine, mais qu'elle peut, dans certains cas, n'être qu'un simple alibi marketing. On souligne l'importance de la cohérence entre règlement technique, pratiques d'élevage et aptitudes des animaux. A quelques exceptions près (race dédiée à une $\mathrm{AOC}$ ), les interactions entre les collectifs de race et de produit demeurent complexes ; les conséquences en matière d'orientation génétique des races en sont discutées. 


\begin{abstract}
The specification of the genetic type within the official rules for animal products under Protected Designations of Origin (PDO): consequences for the breeds.

In France, the integration of the genetic type of animals within the official rules for products under PDO has increased since the nineteennineties. Nowadays it concerns all ewe cheese PDO, the main part of meat PDO and, based on tons yearly produced, about half of cow PDO cheese production and a third of goat PDO cheese production. Regional logics seem to be clear since they express the different concepts of PDO into several marketing chains. In cheese production, we estimate the proportion of dairy cows and dairy ewes contributing to the PDO production to be about $6.5 \%$ and $1 / 3$, respectively. From this point of view, a detailed analysis revealed a great diversity of situations among breeds in given species. Advantages of the couple breed/PDO were analysed. In many cases, the breed contributes to the product's typicity and does enforce the notion of origin but, in some other cases, the breed may only be a marketing argument. The need for consistency of official rules and husbandry with the animal characteristics is pointed out. Except in a few cases where a given breed is devoted to a given PDO, the relations between the breeders' group in charge of the breed management and the group in charge of the product promotion and development are complex. The consequences of such relations on the definition of breeding goals are discussed.
\end{abstract}

LAMBERT-DERKIMBA A., CASABIANCA F., VERRIER E., 2006. L'inscription du type génétique dans les règlements techniques des produits animaux sous AOC : conséquences pour les races animales. INRA Prod. Anim., 19, 357-370. 